\title{
Prognostic Significance of Cofilin Isoforms in Patients With Pancreatic Ductal Adenocarcinoma
}

\author{
Shajedul Islam, Takao Kitagawa and Yasuhiro Kuramitsu * \\ Advanced Research Promotion Center, Health Sciences University of Hokkaido, Hokkaido, Japan
}

Keywords: biomarker, pancreatic ductal adenocarcinoma, cofilin, proteomics, kaplan-meier

To the Editor,

Proteomics is a technique for analyzing protein expression at the cellular level. Proteomic technology that combines two-dimensional gel electrophoresis (2-DE) and liquid chromatography-tandem mass spectrometry (LC-MS/MS) has a high throughput and accuracy [1]. This technique is useful for comprehensively analyzing human disease proteomes and has been widely applied to detect biomarkers and molecular targets in different cancers. We previously performed differential display analysis by using 2-DE and LC-MS/MS between pancreatic cancerous and adjacent non-cancerous tissue samples [2]. One of the numerous spots that showed stronger intensity in cancerous than non-cancerous tissues was identified as non-muscle cofilin (cofilin-1). We further validated the expression of cofilin- 1 by Western blot analysis. We found that cancerous tissues showed significantly higher levels of cofilin-1 than non-cancerous tissues. Meanwhile, muscle cofilin (cofilin-2), an isoform of cofilin, has also been examined in pancreatic cancerous tissue samples. Intriguingly, we observed that cancerous tissues exhibited significantly lower levels of cofilin-2 than non-cancerous tissue samples. This was the first time that cofilin isoforms were differentially expressed in pancreatic cancerous tissues, implying that they could be involved clinicopathologically in patients with PDAC. Therefore, an online bioinformatics tool (https:// kmplot.com/analysis/) has been used to clarify the prognostic significance of cofilin isoforms in PDAC [3]. Using the Kaplan-Meier survival plot, we found a high level of cofilin-1 expression is inversely linked to patient survival. In contrast, high cofilin-2 is positively associated with prolonged patient survival (Figure 1). It is worth mentioning that the results of Kaplan-Meier survival plots were similar to our previous data, which showed that cofilin-1 and cofilin-2 were opposingly expressed in pancreatic cancer tissues. These observations incite interest in cofilin and focus on the relationship between their isoforms and PDAC progression. Together our previous report and the current analysis imply that cofilin isoforms may represent novel potential prognostic biomarkers in patients with PDAC. Further studies are warranted to explore the underlying mechanisms by which cofilin isoforms are involved in patients with PDAC. 


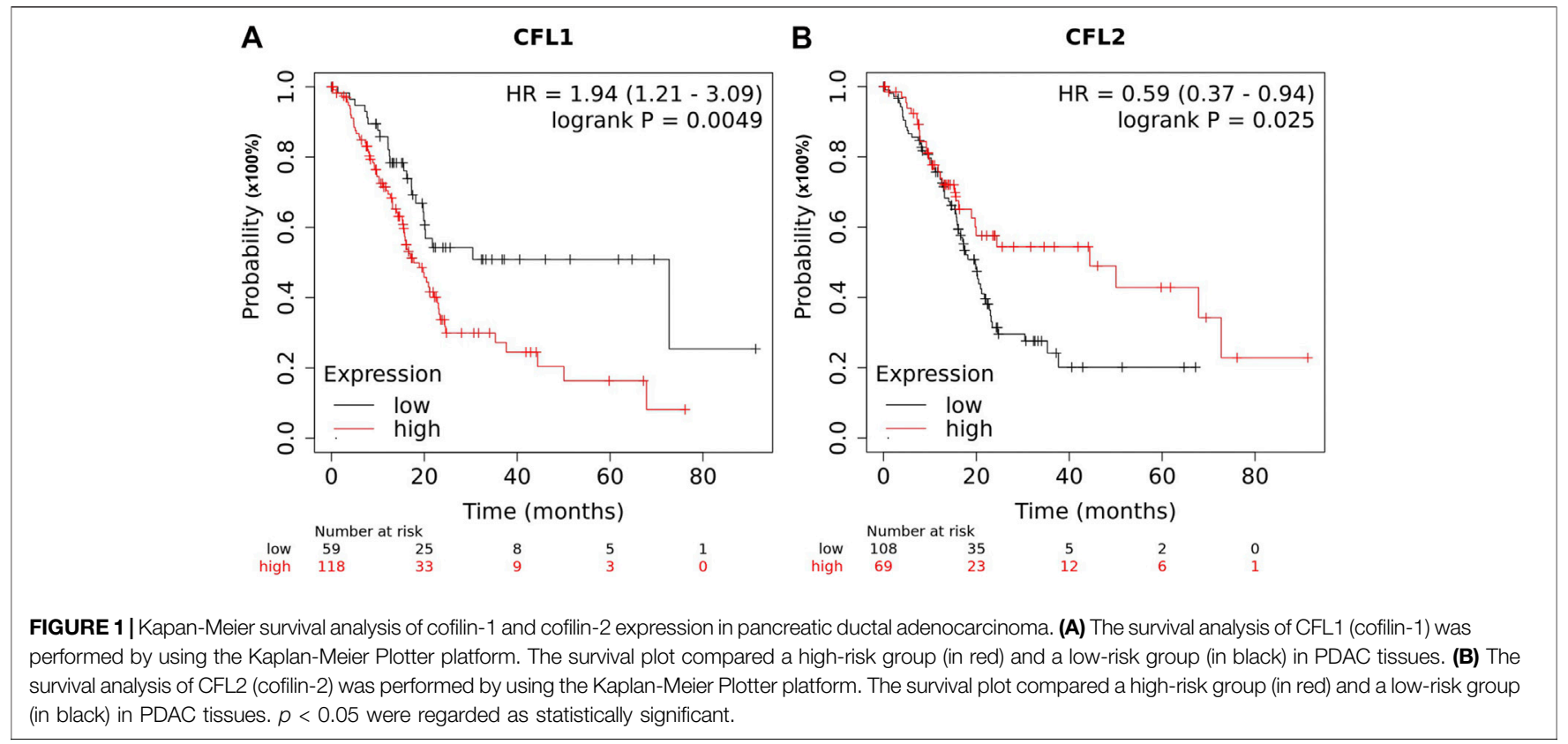

\section{AUTHOR CONTRIBUTIONS}

All authors contributed to the study's conception and design. Data collection and analysis were performed by SI, TK, and YK. SI wrote the first draft of the manuscript, TK and YK commented on previous versions of the manuscript. All authors read and approved the final manuscript.

\section{REFERENCES}

1. Lippolis R, and De Angelis M. Proteomics and Human Diseases. J Proteomics Bioinform (2016) 09:3. doi:10.4172/jpb.1000391

2. Wang Y, Kuramitsu Y, Ueno T, Suzuki N, Yoshino S, Iizuka N, et al. Differential Expression of Up-Regulated Cofilin-1 and Down-Regulated Cofilin-2 Characteristic of Pancreatic Cancer Tissues. Oncol Rep (2011) 26:1595-9. doi:10.3892/or.2011.1447

\section{CONFLICT OF INTEREST}

The authors declare that the research was conducted in the absence of any commercial or financial relationships that could be construed as a potential conflict of interest.

3. Nagy Á, Munkácsy G, and Györffy B. Pancancer Survival Analysis of Cancer Hallmark Genes. Sci Rep (2021) 11:6047. doi:10.1038/s41598-021-84787-5

Copyright (c) 2021 Islam, Kitagawa and Kuramitsu. This is an open-access article distributed under the terms of the Creative Commons Attribution License (CC BY). The use, distribution or reproduction in other forums is permitted, provided the original author(s) and the copyright owner(s) are credited and that the original publication in this journal is cited, in accordance with accepted academic practice. No use, distribution or reproduction is permitted which does not comply with these terms. 